\title{
ON EXTENDING ENDOMORPHISMS TO AUTOMORPHISMS
}

\author{
HILBERT LEVITZ \\ Department of Computer Science \\ Florida State University \\ Tallahassee, FL 32306-4019 \\ WARREN NICHOLS \\ Department of Mathematics \\ Florida State University \\ Tallahassee, FL 32306-3027
}

(Received January 26, 1987 and in revised form March 18, 1987)

ABSTRACT. A monic endomorphism of a structure $A$ can be extended to an automorphism of a larger structure $A^{\star}$. We investigate which properties are preserved by this process.

KEY WORDS AND PHIRASES. Extension of endomorphisms, first-order formulas. 1980 MATHEMATICS SUBJECT CLASSIFICATION CODE, 08 A35.

\section{INTRODUCTION.}

It is known [1, Thm. VII.3.4] that monic endomorphisms of an algebraic structure $A$ can be extended to automorphisms of a larger structure $A^{\star}$ of the same type. In a recent paper [3], Jordan studied the relationship between the two structures in the case in which $A$ is an associative ring. The constructions of $A^{\star}$ in [1] and [3] are quite different.

In this paper, we investigate what can be said in general about the relationship between the properties of $A$ and the properties of $A^{\star}$. Our main result is that all of the "universal formulas" satisfied by the operations and relations on $A$ which are compatible with the given endomorphisms will also be satisfied by the extensions of the operations and relations to $A^{*}$. In comparison to [1], we slightly weaken the hypotheses and broaden the scope of the investigation to include relations, universal first-order formulas, and relationships involving more than one structure. Our results apply to non-algebraic structures, such as metric spaces.

In Section 2, we start with a monoid $M$ acting on a set $A$, and construct a map from $A$ to a "universal" $A^{\star}$ on which $M$ acts by bijections. We show that $A^{*}$ is uniquely determined by certain of its properties. After developing some additional properties, we turn in Section 3 to the question of extending operations and relations from $A$ to $A^{*}$. We also indicate how the analogous procedure may be carried out when 
relationships with other structures ire involvec. We conclude the paper with three examples, showing how our techniques may ti empluyed.

\section{UNIVERSAL M-SETS.}

Recall that a monoid is a set $H$, together with ar associative binary operation $M \times M \rightarrow M$ (which we denote by juxtaposition) and an ideritity element (denoted 1). A (right) $M$-set is a set $A$, tugether with a map $A \times M \rightarrow A$ such that $a l=a$ arid $(a \alpha) B=a(\alpha B)$ for all a $\in A$ and $a, B \in M$. A map of M-sets is a function $\zeta: A \rightarrow B$ on their underlying sets for which $\zeta(a \alpha)=\zeta(a) a$ for all $a \in A, a \in M$. The M-set $A$ is an M-subset of the $M$-set $B$ if $A$ is a subset of $B$ and the inclusion map $A \rightarrow$ $B$ is a map of $M$-sets.

We say that the monoid $M$ acts on the $M$-set $A$ by injections (bijections) if for each $\alpha \in M$ the map $\Theta_{\alpha}^{A}: A \rightarrow A$ given by $a \theta_{\alpha}^{A}=d a$ $(a \in A)$ is irijective (bijective).

A munoid $M_{i}$ is directed by left divisibility if for each $\alpha, 6 \in M$ there exist $\lambda, \mu \in M$ with $\alpha \lambda=\beta \mu$. The monoid $M$ satisfies left cancellation if for $\alpha, \beta, \gamma \in M$ we have that $\gamma \alpha=\gamma \beta$ implies $\alpha=\beta$.

THEOREM 1. Let $M$ be a monoid which is directed by left divisibility and which satisfies left carcellatiur.. Let $A$ be ali li-set. Then there exists an M-set $A^{*}$ and a map $1: A \rightarrow A^{*}$ with the following properties.

(1) $M$ acts on $A^{\star}$ by bijections.

(2) Suppose that $h: A \rightarrow B$ is an $M_{1}$-set map, and $M$ acts on $B$ by bijections. Then there exists a unique $M_{i}$-set map $\zeta: A^{\star} \rightarrow B$ with $\zeta \circ 1=h$.

If $\left(A^{*}\right)^{\prime}$ and $I^{\prime}: A \rightarrow\left(A^{*}\right)^{\prime}$ both satisfy (1) and (2), then there exists a unique isomorphism $\zeta: A^{\star} \rightarrow\left(A^{\star}\right)^{\prime}$ with $\zeta^{\circ} 1=1^{\prime}$.

The M-set $A^{\star}$ (or any isomorphic $\left(A^{*}\right)^{\prime}$, is above) also satisfies

(3) For all $a^{\star} \in A^{\star}$ there exists $\alpha \in M$ and $a \in A$ with $a^{\star} \alpha=1(a)$.

(4) For $a_{1}, a_{2} \in A$ we have $i\left(a_{1}\right)=l_{1}\left(a_{2}\right)$ if and only if there exists $\alpha \in M$ with $a_{1}^{\alpha}=a_{2} \alpha$. In particular, if $M$ acts on $A$ by injections, then $\mathrm{l}$ is injective.

Moreover, if $A^{*}$ and $:: A \rightarrow A^{*}$ satisfy $(1),(3)$, and $(4)$, then they àlso satisfy (2).

PROCF. We first show uniqueness. If $1: A+A^{*}$ and $I^{\prime}: A \rightarrow\left(A^{\star}\right)^{\prime}$ both have properties (1) and (2), then there exist unique $M$-set maps $\zeta$ : $A^{*} \rightarrow$ $\left(A^{*}\right)^{\prime}$ and $\zeta^{\prime}:\left(A^{*}\right)^{\prime} \rightarrow A^{*}$ with $\zeta^{\circ} \mathrm{l}=1^{\prime}$ and $\zeta^{\prime} \circ l^{\prime}=1$. Then $\zeta^{\prime} \circ \zeta^{\circ} \mathrm{l}=1$ and $\zeta^{\circ} \zeta^{\prime} \circ \imath^{\prime}=\imath^{\prime}$. By uniqueness, $\zeta^{\prime} \circ \zeta$ is the identity on $A^{\star}$, and $\zeta^{\circ} \zeta^{\prime}$ is the identity on $\left(A^{\star}\right)^{\prime}$. Thus $\zeta$ is an isomorphism.

Now we construct $A^{\star}$. We first define a relation $\sim$ on $A \times M$ by: $(a, \alpha) \sim(b, \beta)$ if there exist $\lambda, \mu \in M$ with $a \lambda=E \mu$ and $a \lambda=b \mu$. This relation is clearly reflexive and symmetric. Suppose that $(a, a) \sim(b, \beta)$ and $(b, \beta) \sim(c, \gamma)$. We have $\lambda, \mu \in M$ with $\alpha \lambda=\beta \mu$ and $a \lambda=b \mu$, and also $\lambda^{\prime}, \mu^{\prime} \in M$ with $B \lambda^{\prime}=\gamma \mu^{\prime}$ and $b \lambda^{\prime}=c \mu^{\prime}$. Select $\delta, \delta^{\prime} \in M$ with 
$\mu \delta=\lambda^{\prime} \delta^{\prime}$. Then $a \lambda \delta=R \mu \delta=\beta \lambda^{\prime} \delta^{\prime}=\gamma^{\prime} \mu^{\prime} \delta^{\prime}$, and $a \lambda \delta=b \mu \delta=b \lambda^{\prime} \delta^{\prime}=c_{\mu}{ }^{\prime} \delta^{\prime}$, so $(a, a) \sim(c, \gamma)$. Thus $\sim$ is an equivalence relation. We let $A^{*}$ be the set of equiva?ence classes, and denote the equivalence class of $(a, a)$ as $[a, a]$.

Given $(a, \alpha) \in A \times l:$ and $B \in M$, we would like to define $(a, \alpha)_{B} \in A^{*}$ by selecting $\lambda, \mu \in M$ with $\alpha \lambda=\beta_{\mu}$ and setting $(a, \alpha)_{B}=[a \lambda, \mu]$. Suppose that we also have $\alpha \lambda^{\prime}=\beta \mu^{\prime}$. Select $\gamma, \gamma^{\prime} \in M$ with $\mu \gamma=\mu^{\prime} \gamma^{\prime}$. Then $a \lambda \gamma=\beta \mu \gamma=\beta \mu^{\prime} \gamma^{\prime}=\alpha \lambda^{\prime} \gamma^{\prime}$, so $\lambda \gamma=\lambda^{\prime} \gamma^{\prime}$. We now have $\mu \gamma=\mu^{\prime} \gamma^{\prime}$ and $(a \lambda)_{\gamma}=\left(\partial \lambda^{\prime}\right) \gamma^{\prime}$, so $[a \lambda, \mu]=\left[a \lambda^{\prime}, \mu^{\prime}\right]$. Thus our definition of $(a, \alpha)_{\beta}$ does not depend upon the choice of $\lambda, \mu$. Suppose that $(a, \alpha) \sim\left(a^{\prime}, \alpha^{\prime}\right)$. Then there exist $\gamma, \gamma^{\prime} \in M$ with $\alpha \gamma=\alpha^{\prime} \gamma^{\prime}$ and $a \gamma=a^{\prime} \gamma^{\prime}$. Say $\gamma \delta_{1}=\lambda \delta_{2}$ for $\delta_{1}, \delta_{2} \in M$. Then $\alpha^{\prime} \gamma^{\prime} \delta_{1}=\alpha \gamma \delta_{1}=\alpha \lambda \delta_{2}=\beta \mu \delta_{2}$, so $\left(a^{\prime}, \alpha^{\prime}\right)_{B}=\left[a^{\prime} \gamma^{\prime} \delta_{1}, \mu \delta_{2}\right]=$ $\left[a \gamma \delta_{1}, \mu \delta_{2}\right]=\left[a \gamma \delta_{2}, \mu \delta_{2}\right]=[a \lambda, \mu]=(a, \alpha)_{B}$. Thus there is a well-defined function $A^{\star} \times M \rightarrow A^{\star}$ given by $[a, \alpha] B=[a \lambda, \mu]$, where $\alpha \lambda=\beta \mu$.

Clearly $[a, \alpha] 1=[a, \alpha]$. Given $[a, \alpha] \in A^{\star}$ and $B_{1}, \beta_{2} \in M$, we have $[a, \alpha]_{1}=\left[a \lambda_{1}, \mu_{1}\right]$ where $\alpha \lambda_{1}=\beta_{1} \mu_{1}$, and $\left[a \lambda_{1}, \mu_{1}\right] \beta_{2}=\left[a \lambda_{1} \lambda_{2}, \mu_{2}\right]$ where ${ }_{\mu_{1} \lambda_{2}}=\beta_{2} \mu_{2}$. Then $\alpha \lambda_{1} \lambda_{2}={ }{ }_{1}{ }_{1} \lambda_{2}{ }_{2}=\beta_{1} \beta_{2} \mu_{2}$, so $[a, \alpha]_{\beta_{1} \beta_{2}}=\left[a \lambda_{1} \lambda_{2}, \mu_{2}\right]=$ $\left([a, \alpha]_{B_{1}}\right)_{B_{2}}$. Thus $A^{*}$ is an $M$-set. Since for $[a, \alpha] \in A^{\star}$ and $B \in M$ we have $[a, \alpha]=[a, b \alpha]_{B}$, the action of each $B \in M$ on $A^{*}$ is surjective. Suppose that $\left[a_{1}, \alpha_{1}\right]_{B}=\left[a_{2}, \alpha_{2}\right]_{B}$. Then $\left[a_{1} \lambda_{1}, \mu_{1}\right]=\left[a_{2} \lambda_{2}, \mu_{2}\right]$, where $\alpha_{1} \lambda_{1}=B \mu_{1}$ and $\alpha_{2} \lambda_{2}=B \mu_{2}$. So there exists $\gamma_{1}, \gamma_{2} \in M$ with $\mu_{1} \gamma_{1}=\mu_{2} \gamma_{2}$ and $a_{1} \lambda_{1} \gamma_{1}=a_{2} \lambda_{2} \gamma_{2}$. Since $\alpha_{1} \lambda_{1} \gamma_{1}={ }_{\star}^{B \mu_{1} \gamma_{1}}=\beta_{1} \mu_{2} \gamma_{2}=\alpha_{2} \lambda_{2} \gamma_{2}$, we have $\left[a_{1}, \alpha_{1}\right]=\left[a_{2}, \alpha_{2}\right]$. Thus $M$ acts on $A^{*}$ by bijections, and we have shown (1).

The map $1: A \rightarrow A^{*}$ given by $\mathfrak{l}(a)=[a, 1]$ is clearly a map of $M$-sets. Note that for $a^{\star}=[a, \alpha] \in A^{*}$ we have $a^{\star} \alpha=[a, \alpha] \alpha=[a, 1]=\imath(a)$, verifying (3). For $a_{1}, a_{2} \in A$ we have,$\left(a_{1}\right)=i\left(a_{2}\right)$ if and only if there exists $\alpha_{1}, \alpha_{2} \in M$ with $l_{\alpha_{1}}=1 \alpha_{2}$ and $a_{1} \alpha_{1}=a_{2} \alpha_{2}$. Since we must have $\alpha_{1}=\alpha_{2}$, we have verified (4).

Let us now assume that we are given $A^{\star}$ and $: A \rightarrow A^{\star}$ satisfying (1), (3), and (4). Let $h: A \rightarrow B$ be an M-set map from $A$ to an M-set $B$ on which $M$ acts by bijections. Suppose that there exists an $M$-set map $\zeta: A^{\star} \rightarrow B$ with $\zeta_{\circ}=h$. Let $a^{\star} \in A^{\star}$. Select $a \in M$ and $a \in A$ with $a^{\star} \alpha={ }_{l}(a)$. Then $\zeta\left(a^{\star}\right)_{\alpha}=\zeta\left(a^{\star} \alpha\right)=\zeta(l(a))=h(a)$, so $\zeta\left(a^{*}\right)=h(a)\left(\theta_{\alpha}^{B}\right)^{-1}$. Thus $\zeta$, if it exists, is unique. To obtain existence, we must show that for $a^{*} \in A^{*}$ the expression $\zeta\left(a^{\star}\right)=h(a)\left(\theta_{\alpha}^{B}\right)^{-1}$ does not depend upon the choice of $\alpha \in M$ and $a \in A$ with $a^{\star} \alpha=1(a)^{\alpha}$, and yields an M-set map with $\zeta \circ_{1}=h$.

Let us first note that if $l_{1}\left(a_{1}\right)={ }_{l}\left(a_{2}\right)$ then by (4) we can find $\beta \in M$ with $a_{1} \beta=a_{2} B$, and so $h\left(a_{1}\right)=h\left(a_{1}\right)_{B}\left(\theta_{B}^{B}\right)^{-1}=h\left(a_{1} \beta\right)\left(\theta_{B}^{B}\right)^{-1}=h\left(a_{2} B\right)\left(\theta_{B}^{B}\right)^{-1}=$ $h\left(a_{2}\right)_{B}\left(\theta_{B}^{B}\right)^{-1}=h\left(a_{2}\right)$. Now let $a^{\star} \in A^{\star}$, and suppose we have $\alpha_{1}, \alpha_{2} \in M$ and $a_{1}, a_{2} \in A$ with $a^{*} \alpha_{1}={ }_{1}\left(a_{1}\right)$ and $a^{\star} \alpha_{2}={ }_{1}\left(a_{2}\right)$. Select $\lambda_{1}, \lambda_{2} \in M$ with $\alpha_{1} \lambda_{1}=\alpha_{2} \lambda_{2}$. Then ${ }_{1}\left(a_{1} \lambda_{1}\right)=a^{*} \alpha_{1} \lambda_{1}=a^{*} \alpha_{2} \lambda_{2}={ }_{1}\left(a_{2} \lambda_{2}\right)$, so $h\left(a_{1} \lambda_{1}\right)=$ $h\left(a_{2} \lambda_{2}\right)$. Thus $h\left(a_{1}\right)\left(\theta_{\alpha_{1}}^{B}\right)^{-1}=h\left(a_{1}\right) \lambda_{1}\left(\theta_{\lambda_{1}}^{B}\right)^{-1}\left(\theta_{\alpha_{1}}^{B}\right)^{-1}=h\left(a_{1} \lambda_{1}\right)\left(\theta_{\alpha_{1} \lambda_{1}}^{B}\right)^{-1}=$ 
$h\left(a_{\alpha_{2} \lambda_{2}}\right)\left(\theta_{u_{2} \lambda_{2}}^{B}\right)^{-1}=h\left(a_{2}\right)\left(\Theta_{\alpha_{2}}^{B}\right)^{-1}$, so the function $\zeta: A^{*} \rightarrow B$ is well-defined.

Let ${ }^{4}{ }_{a}^{\lambda} \star^{2} \in A^{\star}, B \in M_{1}{ }^{\alpha}$. Select $a \in M, a \in A$ with $a^{\star} \alpha=i(a)$. Select $\lambda, \mu \in M$ with $\alpha \lambda=B_{\mu}$. Note that $\theta_{a}^{B} \theta_{\lambda}^{B}=\theta_{B}^{B} \theta_{\mu}^{B}$, so $\theta_{\lambda}^{B}\left(\theta_{\mu}^{B}\right)^{-1}=\left(e_{\alpha_{B}^{B}}^{B}\right)^{-1} \theta_{\theta^{B}}^{B}$. Since $\left(a^{\star} B\right)_{\mu}=a^{\star} \alpha \lambda=i(a \lambda)$, we have $\zeta\left(a^{\star} B\right)=h(a \lambda)\left(\theta_{\mu}^{B}\right)^{-1}=h(a) \theta_{\lambda}{ }^{B}\left(\theta_{\mu}^{B}\right)^{-1}=$ $h(a)\left(\theta_{\alpha}^{B}\right)^{-1} \theta_{B}^{B}=\zeta\left(a^{*}\right) e$. Thus $\zeta$ is an M-set map. Clearly $\zeta_{01}=h$, and we are done.

Let $M$ be a monoid which is directed by left divisibility and which satisfies left cancellation. Let $M$ denote the category of $M$-sets, arid let $: i^{*}$ denote the category of $M$-sets on which $M$ acts by bijections. We associate $A^{*} \in O b\left(N^{\star}\right)$ to $A \in O b(N)$. Let ${ }^{\prime} A: A \rightarrow A^{*}$ be the map given by Theorem. 1 . Now let $g: A \rightarrow B$ be any $M$-set map. Since ${ }^{\prime} B^{\circ} g$ is a map from $A$ to an $M$-set $B^{*}$ on which $M$ acts by bijections, we have by Theorem 1 that there is a unique map $g^{*}: A^{\star} \rightarrow B^{*}$ with $g^{*}{ }^{\circ} A={ }^{\circ} B^{\circ g}$. If $h: B+C$ is another M-set map, then $h^{*} \circ g^{*}{ }^{*}{ }_{1} A=h^{*} \circ{ }^{1} B^{\circ} g={ }^{1} C^{\circ h \circ g}$, so $h^{*} \circ g^{*}=(h \circ g)^{*}$ by uniqueness. Thus we have the following.

THEOREM 2. Let $M$ be a monoid which is directed by left divisibility and which satisfies left cancellation. Then there is a functor () ${ }^{*}$ from the category $M_{1}$ of $M$-sets to the category $M^{*}$ of $M$-sets on which $M$ acts by bijections, which associates to each $M$-set $A$ the "universal" $M$-set $A^{\star}$ on which $M$ acts by bijections, and to each $M$-set map $g: A \rightarrow B$ the unique M-set map $g^{\star}: A^{\star} \rightarrow B^{\star}$ for which $g^{\star} \circ{ }^{\prime} A={ }^{1} B^{\circ g}$.

We shall refer to $g^{\star}$ as the extension of $g$.

In the next section, we will need an additional property of the functor ()$^{\star}$. Let $A_{1}, \ldots, A_{r}$ be $M$-sets. Then $A_{1} \times \ldots \times A_{r}$ is an $M$-set, with $M$ acting via $\left(a_{1}, \ldots, a_{r}\right) \alpha=\left(a_{1} \alpha, \ldots, a_{r} \alpha\right)$ for $\left(a_{1}, \ldots, a_{r}\right) \in A_{1} \times \ldots \times A_{r}$ and $\alpha \in M$. This $M_{\text {-set }}$ is the product of $A_{1}, \ldots,{ }_{\star}{ }_{r}$ in the category $M$. It is easy to see that $M$ acts by bijections on $A_{1^{*}}^{*} \ldots \times A_{r}^{*}$, and thus that $A_{1}^{\star} \times \ldots \times A_{r}^{\star}$ is the product of $A_{1}^{*}, \ldots, A_{r}^{*}$ in $M^{\star}$.

PROPOSITION 3. The functor ()$^{\star}$ preserves finite products. More explicitly, for any M-sets $A_{1}, \ldots, A_{r}$, the unique map $\zeta$ from $\left(A_{1} \times \ldots \times A_{r}\right)^{*}$ to $A_{1}^{*} \times \ldots \times A_{r}^{*}$ such that $\zeta^{\circ}{ }^{1} A_{1} \times \ldots \times A^{\prime}={ }^{1} A_{A} \times \ldots \times 1{ }^{A}$ is an isomorphism.

PROOF. By Theorem 1, it Suffices to show that ${ }^{r} A_{1}^{\star} \times \ldots \times A_{r}^{*}$ and ${ }^{2} A \times \ldots \times{ }^{1} A_{r}$ satisfy (1), (3), and (4). We have already observed that it is easy to see ${ }^{2}$ that (1) holds. Given $\left(a_{1}^{*}, \ldots, a_{r}^{*}\right) \in A_{1}^{*} \times \ldots \times A_{r}^{*}$, there exist $\alpha_{j} \in M$ and $a_{j} \in A_{j}(j=1, \ldots, r)$ with $a_{j} \alpha_{j}={ }^{2} A_{j}\left(a_{j}\right)$, each $j$. Select ${ }_{\gamma, \lambda_{1}}, \ldots, \lambda_{r} \in M$ so that $\alpha_{j^{\lambda} j}=\gamma$, all ${ }_{j}$. Then $a_{j \gamma}^{*}={ }^{2} A_{j}\left(a_{j}{ }_{j}\right)$, all $j$, so $\left(a_{1}^{*}, \ldots, a_{r}^{*}\right)_{r}=\left({ }_{1} A_{1} \times \ldots \times{ }_{1} A_{r}\right)\left(a_{1} \lambda_{1}, \ldots, a_{r} \lambda_{r}\right)$, and we have verified (3). For $\left(a_{1}, \ldots, a_{r}\right),\left(a_{1}^{\prime}, \ldots, a_{r}^{1}\right)^{r} \in A_{1} \times \ldots \times A_{r}$ we have ${ }^{1} A_{1}{ }^{\times} \ldots \times{ }^{1} A_{r}\left(\left(a_{1}, \ldots, a_{r}\right)\right)={ }^{1} A_{A} \times \ldots \times{ }^{1} A_{r}\left(\left(a_{1}, \ldots, a_{r}^{1}\right)\right)$ if and only if there exist $\alpha_{1}, \ldots, \alpha_{r} \in M$ with $a_{j} \alpha_{j}=a_{j} \alpha_{j}, j \stackrel{A_{r}}{=}, \ldots, r$. In this case we can find $a$ single $\gamma=\alpha_{1} \lambda_{1}=\ldots=\alpha_{r}{ }_{r}$ with $a_{j \gamma}=a_{j \gamma}^{\prime}$, all $j$, so $\left(a_{1}, \ldots, a_{r}\right)_{\gamma}=\left(a_{1}^{1}, \ldots, a_{r}^{\prime}\right)_{r}$. As the reverse implication is trivial, we have verified (4), and we are done.

Let us also observe that for any M-set $A$ and any $r>0$ there is an 
M-set map $\delta_{A}^{(r)}: A \rightarrow A^{(r)}$ (where $A^{(r)}$ denotes the product of $r$ copies of A) given by $\delta_{A}^{(r)}(a)=(a, \ldots, a)$ for all a $\in A$, and that we have $\left(\delta_{A}^{(r)}\right)^{\star}=$ $\delta_{A^{\star}}^{(r)}$.

\section{EXTENSION OF OPERATIONS AND RELATIONS}

In this section, we show how the results of Section 2 can be used to extend operations, functions, and relations from $A$ to $A^{*}$.

We refer to elements of $A$ as constants. A constant $C$ is admissible if $c_{\alpha}=c$ for all $\alpha \in M$. Note that there is a trivial $M$-set $\{\cdot\}$, for which $\cdot \alpha=$. for all $\alpha \in M$, and that we may view an admissible constant of $A$ as being the same thing as an M-set map from $\{\cdot\}$ to $A$. For $r>0$, an $r$-place operation on $A$ is a function $f: A(r) \rightarrow A$. We say that the operation is admissible if it is a map of M-sets. More generally, an $r$-place function on $A$, with values in the set $B$, is a function $f: A(r) \rightarrow B$. The function $f$ is admissible if $B$ is an M-set, and $f$ is a map of M-sets. (Any set $B$ may be given the trivial M-structure, with $b a=b$ for all $b \in B, a \in M$.) An $r$-place relation on $A$ is a subset $R$ of $A^{(r)}$. We may view $R$ as being a function $R: A(r) \rightarrow T$, where $T=\{0,1\}$ is our set of "truth values". Then for $a=\left(a_{1}, \ldots, a_{r}\right) \in A^{(r)}$, we say that $a \in R$ iff $R(a)=1$. The relation $R$ is admissible if for all $a \in A^{r}$ and $a \in M$, we have $a \in R$ if and only if $a \alpha \in R$. If we consider $R$ as a function with values in the set $T$, with $T$ given the trivial M-structure, then this condition is simply that $R$ be an admissible function, that is, an M-set map.

We see that admissible operations, functions, and relations on $M$-sets may be viewed as $M$-set maps. By Theorem 2, each has a unique extension from $M$ to $M^{*}$. Moreover, any relationship between certain $M$-sets which may be expressed as saying that a certain composite of M-set maps into the M-set $T$ has constant value 1 will hold when we pass from $M$ to $M^{*}$; by Proposition 3 , a composite defined in terms of a product in $M$ will be defined in the corresponding manner in $M^{\star}$.

This result is quite powerful. We shall work out some consequences for the case in which a single M-set is involved.

We shall describe what we mean by a "universal formula" for an M-set A. The basic building blocks for the formula are the admissible constants of $A$ and the variables $x_{1}, x_{2}, \ldots$. The formula will be a finite expression; let us say that the variables that occur in it are among $x_{1}, \ldots x_{n}$. We first explain, recursively, what we mean by a term of the formula. Each term is an M-set map from $A^{(n)}$ to $A$. A constant $c$ is the map sending a $\in A^{(n)}$ to $c \in A$. A variable $x_{i}$ is the map sending $\left(a_{1}, \ldots, a_{n}\right) \in A(n)$ to $a_{i} \in A$. If $t_{1}, \ldots, t_{r}$ are terms and $f$ is an $r$-place operation on $A$, then $f\left(t_{1}, \ldots, t_{r}\right)$ is the term sending $a \in A^{(n)}$ to $f\left(t_{1}(a), \ldots, t_{r}(a)\right) \in A$. We next explain, recursively, how to create formulas. Each formula will be an $M$-set map from $A^{(n)}$ to the trivial M-set $T=\{0,1\}$. If $R$ is an r-place relation on $M$ and $t_{1}, \ldots, t_{r}$ are terms, then the composite $R\left(t_{1}, \ldots, t_{r}\right)$, 
defined by $R\left(t_{1}, \ldots, t_{r}\right)(a)=R\left(t_{1}(a), \ldots, t_{r}(a)\right)$ for $a \in A(n)$, is a formula. If $A$ is a formula, then $\left({ }_{7} A\right)$ is the formula given by $\left({ }_{7} A\right)(a)=1$ if $A(a)=0,\left({ }_{7} A\right)(a)=0$ if $A(a)=1$. Similarly, if $A$ and $B$ are formulas, then $(A \vee B),(A \wedge B),(A \rightarrow B),(A \leftrightarrow B)$ are the formulas obtained by viewing the logical operations " $v$ "(or), "A" (and), " $\rightarrow$ " (implies), and " $\leftrightarrow$ " (iff) as M-set maps $L: T \times T \rightarrow T$, and composing with the map $A^{(n)} \rightarrow T \times T$ sending a to $(A(a), B(a))$. Note that this latter map is $(A \times B) \circ \delta_{A}^{(2)}(n)$.

Thus a universal formula for $A$, involving variables from $x_{1}, \ldots, x_{n}$, is interpreted as an M-set map from $A(n)$ to $T$. We say that the formula "holds" if, as a function, it is constant with value 1 . We call the formula "universal" because, formally, we interpret it as holding if and only if it holds "with a universal quantifier for each variable that appears."

By Theorem 2, Proposition 3, and the observation following Proposition 3, we have the following.

THEOREM 4. Let $M$ be a monoid directed by left divisibility and satisfying left cancellation. Let $A$ be an M-set. Then every admissible operation and relation on $A$ has a unique extension to $A^{*}$. These extensions satisfy the same universal formulas in $A^{*}$ that were satisfied by the original operations and relations in $A$.

Let us now discuss the uses of this result, as well as its limitations.

The most important limitation has to do with the "equals" relation. We often want to express the idea that two composites are equal. For example, if " + " is a binary operation on $A$, then the formula $x_{1}+x_{2}=x_{2}+x_{1}$ means that $=\left(\left(a_{1}+a_{2}\right),\left(a_{2}+a_{1}\right)\right)$ is 1 for all $a_{1}, a_{2} \in A$. In order to be able to apply the theorem, = must be an admissible relation.

PROPOSITION 5. The equals relation is admissible iff $M$ acts on $A$ by injections. In this case, $=^{\star}$ is the equals relation on $A^{\star}$.

PROOF. For equals to be admissible we require $a_{1}=a_{2}$ iff $a_{1} \alpha=a_{2} \alpha$, for all $a_{1}, a_{2} \in A, \alpha \in M$. This is clearly the condition that $M$ act on $A$ by injections. In this case, suppose that $a_{1}^{\star}{ }^{\star} a_{2}^{*}$ for $a_{1}^{\star}, a_{2}^{\star} \in A^{*}$. There exists $\gamma \in M$ with $a_{1^{*}}^{\star} \in A$ and $a_{f^{*}}^{\star} \in A_{*}$. (This makes sense, since ${ }^{2} A$ is injective.) Then $a_{1}^{\star} \gamma=a_{2}^{\star} \gamma$, so $a_{1}^{*}=\left(a_{1}^{\star} \gamma\right)\left(\theta_{\gamma}^{A^{*}}\right)^{-1}=\left(a_{2}^{*}\right)\left(\theta_{\gamma}^{A^{*}}\right)^{-1}=a_{2}^{*^{*} A}$.

When $M$ acts on $A$ by injections, then from Theorem 4 and Propostion 5 we can often get that $A^{*}$ is the same type of algebraic object as $A$. For example, the operation + on $A$ is associative iff it satisfies $\left(x_{1}+x_{2}\right)+x_{3}$ $=x_{1}+\left(x_{2}+x_{3}\right)$, and in this case $\left(x_{1}+{ }^{*} x\right)+{ }^{*} x_{3}=x_{1}+{ }^{*}\left(x_{2}+{ }^{\star} x_{3}\right)$, so $+{ }^{*}$ is an associative operation on $A^{*}$.

Sometimes some reformulation is required. Suppose that $A$ is a field, and that $M$ acts injectively on $A$ as field endomorphisms. For a $\in A$, set $v(a)=0$ if $a=0, v(a)=a^{-1}$ if $a \neq 0$. Then $v$ is an admissible operation on $A$, and $A$ satisfies the formula $\left(x_{1}=0\right) \vee\left(x_{1} \cdot v\left(x_{1}\right)=1\right)$. Since $A^{\star}$ satisfies the same formula, we obtain that $A^{*}$ is a field.

Our next result provides another method for obtaining properties of $A^{\star}$ in terms of properties of $A$. 
Let $f$ be an admissible $r$-place operation on $A$, and let $f^{\star}$ be its extension to $A^{\star}$. We first note that $f^{\star}$ is defined on $I(A)$ by $f_{*}^{*}\left(l_{1}\left(a_{1}\right), \ldots, i\left(a_{r}\right)\right)=i f\left(a_{1}, \ldots, a_{r}\right)$ for $a l l\left(a_{1}, \ldots, a_{r}\right) \in A^{(r)}$. Now let $a_{1}^{*}, \ldots, a_{r}^{*}$ be elements of $A^{*}$. There exists an element $r$ of $M$ and elements $a_{1}, \ldots, a_{\star} r$ of $A$ with $a_{j^{*}}^{*}=1\left(a_{j_{A \star}}\right)$ for $j=1, \ldots, r$. We have $f^{\star}\left(a_{1}^{\star}, \ldots, a_{r}^{*}\right)=f^{\star^{r}}\left({ }_{1}\left(a_{1}\right)\left(\theta_{\gamma_{A^{\star}} A^{\star}}\right)^{-1}, \ldots,\left(_{1}\left(a_{r}\right)\left(\theta_{\gamma}^{A^{\star}}\right)^{-1}\right)=f^{\star}\left({ }_{1}\left(a_{1}\right), \ldots, a_{1}\left(a_{r}\right)\right)\right.$ $\left(\theta_{\gamma}^{A^{*}}\right)^{-1}=\left({ }_{i} f\left(a_{1}, \ldots, a_{r}\right)\right)\left(\theta_{\gamma}^{\gamma} A^{\star}\right)^{-1}$. Since the same argument goes through for relations, we have the following result.

PROPOSITION 6. Let $M$ be a monoid divided by left divisibility and satisfying left cancellation. Let $A$ be an M-set. Then $A^{*}$ is the directed union of the sets $l(A)\left(\theta_{\gamma}^{A^{\star}}\right)^{-1}, \gamma \in M$. The admissible operations and relations on $A$ are extended from ${ }_{l}(A)$ to ${ }_{l}(A)\left(\theta_{\gamma}^{A^{\star}}\right)^{-1}$ via $\theta_{\gamma}^{A^{\star}}$; thus $i(A)\left(\theta_{\gamma}^{A^{\star}}\right)^{-1}$ is "isomorphic" to ${ }^{2}(A)$.

Note, however, that ${ }^{\prime}(A)\left(\theta_{\gamma}^{A^{\star}}\right)^{-1}$ may not be closed under the action of

$M$ if $M$ is not commutative.

We now present our three examples. Note that [1, Thm. VII.3.4] does not apply in these cases, either because the "locally $K$ " conclusion of that theorem does not allow us to infer that certain relations and formulas extend from $A$ to $A^{*}$, or because we need to consider two M-sets simultaneously, together with a map between them.

EXAMPLE 7. Let $N$ denote the monoid of natural numbers under addition. Let $A$ be the set $N \times N$. Define a relation < on $A$ by: $\left(n_{1}, n_{2}\right)<\left(m_{1}, m_{2}\right)$ iff $n_{1}+n_{2}<m_{1}+m_{2}$. Then < is a strict order on $A$ - that is, it satisfies the universal formulas $7\left(x_{1}<x_{1}\right) ;\left(x_{1}<x_{2}\right) \rightarrow{ }_{7}\left(x_{2}<x_{1}\right)$; and $\left(\left(x_{1}<x_{2}\right) \wedge\left(x_{2}<x_{3}\right)\right)$ $\rightarrow\left(x_{1}<x_{3}\right)$. We give $A$ an $N$-set structure by $\left(n_{1}, n_{2}\right) \cdot n=\left(0, n_{1}+n_{2}+n\right)$ for all $\left(n_{1}, n_{2}\right) \in A, n \in N$. The relation $<$ is admissible. By Theorem 4 , the universal $N$-set $A^{*}$ has a strict order $<$ which extends $<$. Let the set $\mathbb{Z}$ of integers be an $N$-set under addition. Define $j: A \rightarrow \mathbb{Z}$ by $j\left(\left(n_{1}, n_{2}\right)\right)$ $=n_{1}+n_{2}$. Then $j$ is an $N$-set map, with $Z$ and $j$ satisfying (1), (3), and (4) of Theorem 1, so we may take $A^{*}$ and, to be $Z$ and $j$. We see that $<$ is the usual order on $\mathbf{Z}$.

EXAMPLE 8. Let $A, N$ be as above. Change the action of $N$ on $A$ to $\left(n_{1}, n_{2}\right) \cdot n=\left(n_{1}+n, n_{2}+n\right)$ for $\left(n_{1}, n_{2}\right) \in A, n \in N$. Then < is still an admissible relation, and now $N$ acts on $A$ by injections. Thus we can view $A^{*}$ as being a set containing $A$. We can equip $A$ with the metric $d: A \rightarrow \mathbb{R}$ given by $d\left(\left(n_{1}, n_{2}\right),\left(n_{1}^{\prime}, n_{2}^{\prime}\right)\right)=\sqrt{\left(n_{1}-n_{1}^{\prime}\right)^{2}+\left(n_{2}-n_{2}^{\prime}\right)^{2}}$ for $\left(n_{1}, n_{2}\right),\left(n_{1}^{\prime}, n_{2}^{\prime}\right)$ $\in A$. We give $\mathbb{R}$ the trivial $N$-set structure; then $d$ is a map of $N$-sets. That $d$ is a metric is expressed by the universal formulas: $d\left(x_{1}, x_{2}\right) \geq 0$; $\left(d\left(x_{1}, x_{2}\right)=0\right) \leftrightarrow\left(x_{1}=x_{2}\right) ; d\left(x_{1}, x_{2}\right)=d\left(x_{2}, x_{1}\right) ; d\left(x_{1}, x_{3}\right) \leq d\left(x_{1}, x_{2}\right)$ $+d\left(x_{1}, x_{2}\right)$. Each formula may be interpreted as asserting that a certain composite of $N$-set maps from $A^{(2)}$ (or $A^{(3)}$ ) to $T=\{0,1\}$ has the constant value 1. By Theorem 2 and Proposition 3, the corresponding result holds for $A^{*}$. Thus we have that $A^{*}$ is a metric space containing $A$, equipped with a strict order $<^{\star}$, on which $N$ acts by isometric automorphisms. Using Theorem 
1 we see that $A^{*}$ can be realized as $\mathbb{Z} \times \mathbb{Z}$, with $d^{*}$ and $<^{*}$ given by the formal extension to $\mathbb{Z} \times \mathbb{Z}$ of the formulas defining $d$ and < respectively.

EXAMPLE 9. Let $F$ be a field, and let $K=F\left(x_{1}, \ldots x_{t}\right)$ be the field of rational functions over $F$ in $t$ indeterminates. Let $\theta: K \rightarrow K$ be the $F$-algebra homomorphism sending $x_{i}$ to $x_{i}^{2}$, for $1 \leqq i \leqq t$, and give $k$ the $N$-set structure $(k, n) \rightarrow k^{n}$ for $k \in K, n \in N$. Let $G=\mathbb{R} \cup\{\infty\}$. Extend the operation " + " and the relation "<" from $\mathbb{R}$ to $G$ by setting $a+\infty=\infty=\infty+$ $a=\infty+\infty$ and $a<\infty$ for all a $\in \mathbb{R}$. Make $G$ into an $N$-set via $(a, n) \rightarrow a 2^{n}$ for $a \in \mathbb{R}, n \in N$ and $(\infty, n) \rightarrow \infty$. Let $\alpha_{1}, \ldots, \alpha_{t}$ be rationally independent elements of $R$. Define a function $\nu: K \rightarrow G$ as follows: $\nu(0)=\infty ; \nu(m)$, where $m=a x_{1}^{e} 1 \ldots x_{t}^{e_{t}}$ is a nonzero monomial $(0 \neq a \in F)$, is $\quad e_{1}^{\alpha}{ }_{1}+\ldots+e_{t^{\alpha}}$; $\nu(f)$, where $f=m_{1}+\ldots+m_{s}$ is the unique representation of $0 \neq f \epsilon$ $F\left[X_{1}, \ldots, x_{t}\right]$ as a sum of distinct monomials $m_{j}$, is inf $\left\{v\left(m_{j}\right)\right\}_{i=1}^{s}$, and $v(f / g)=$ $v(f)-v(g)$ for $0 \neq f, g \in F\left[x_{1}, \ldots, x_{t}\right]$. The function $v$ satisfies $v(x y)=$ $v(x)+v(y)$ for all $x, y \in K$, and $v(x+y) \leqq$ inf $\{v(x), v(y)\}$ for all $x, y \in K$ [2, Theorem 18.3]; that is, $v$ is a valuation on the field $k$. It is easy to see that $\nu$ is a niap of $\mathrm{N}$-sets. We note that the ring operations on $\mathrm{K}$ are admissible, and that,$+<$, and inf are admissible on G. Arguing as in Example 8 , we see that $N$ acts as automorphisms of an extension field $k^{\star}$ of $K$. Since $N$ already acts bijectively on $G$, we see that $G^{\star}=G$, and that $\nu^{\star}: K^{\star} \rightarrow G$ is a valuation on $k^{*}$. The structure of $K^{*}$ is tolerably complicated; it is an infirite algebraic extension of $K$.

\section{REFERENCES}

1. COHN, P.M., Universal Algebra, Harper and Row, New York, 1965.

2. GILMER, R., Multiplicative Ideal Theory, Dekker, New York, 1972.

3. JORDAN, D.A., Bijective extensions of injective ring endomorphisms, J. London Math. Soc. (2), 25 (1982), 435-448. 


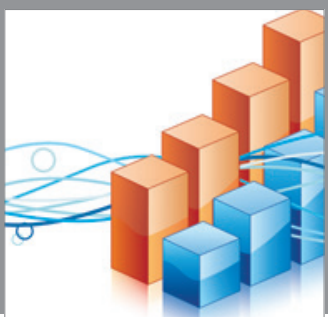

Advances in

Operations Research

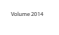

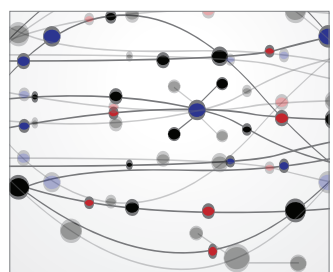

\section{The Scientific} World Journal
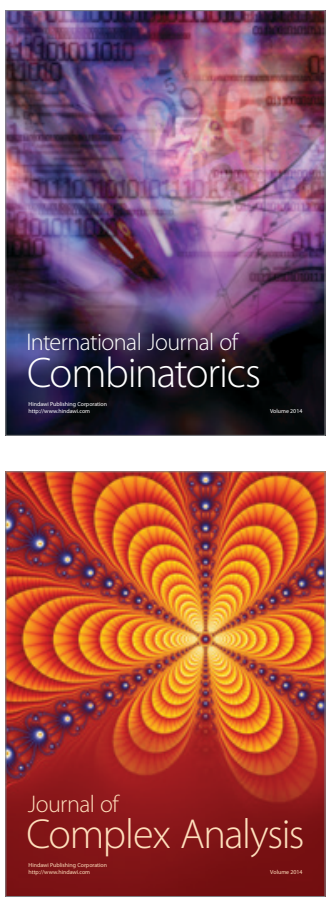

International Journal of

Mathematics and

Mathematical

Sciences
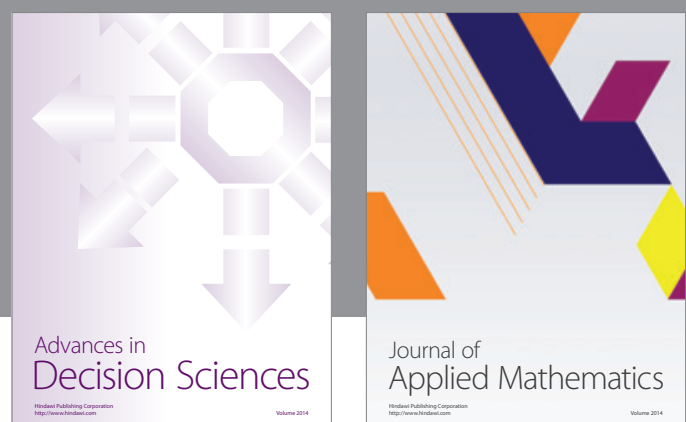

Journal of

Applied Mathematics
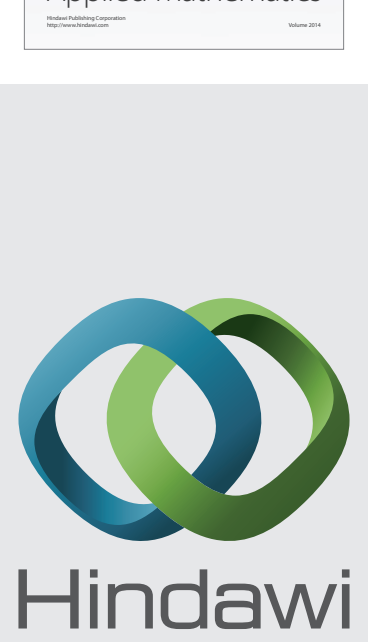

Submit your manuscripts at http://www.hindawi.com
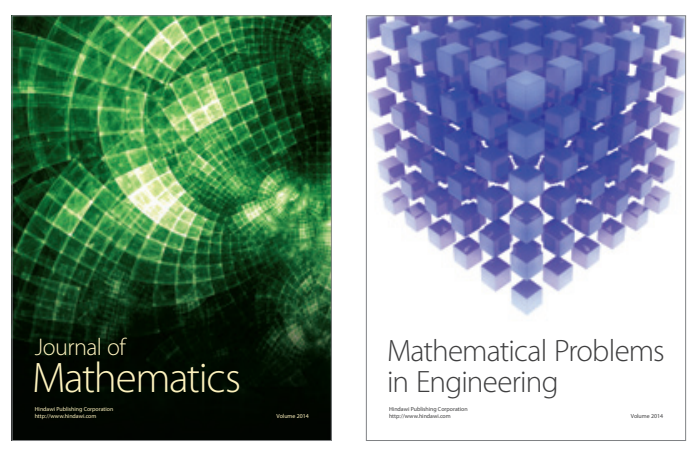

Mathematical Problems in Engineering
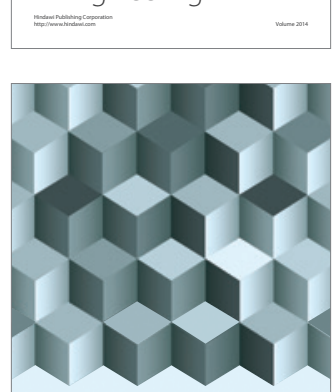

Journal of

Function Spaces
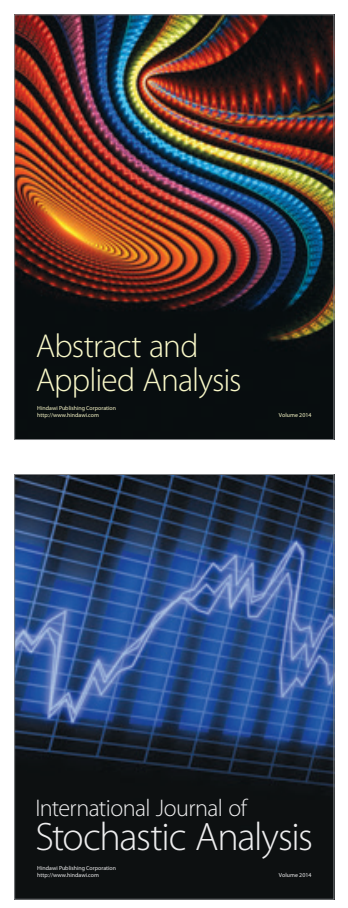

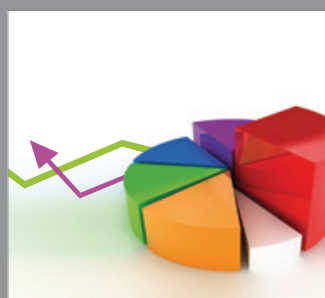

ournal of

Probability and Statistics

Promensencen
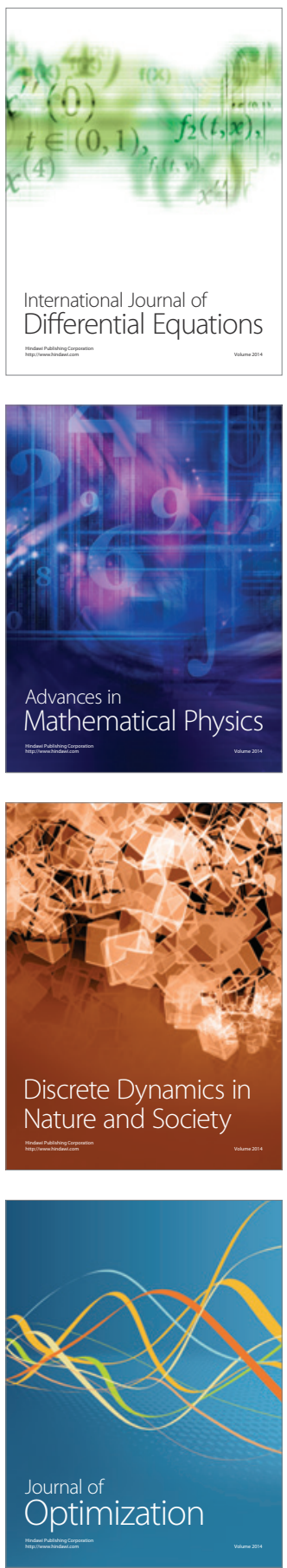